\title{
Mycobacterium bovis BCG infection alters the macrophage N-glycome
}

Clément Delannoy ${ }^{\mathrm{a}}$, Chin Huang ${ }^{\mathrm{b}, \mathrm{c}}$, Bernadette Coddeville ${ }^{\mathrm{a}}$, Jian-You Chen ${ }^{\mathrm{b}}$, Dounia Mouajjah ${ }^{\mathrm{a}}$, Sophie Groux-Degroote ${ }^{\mathrm{a}}$, Anne Harduin-Lepers ${ }^{\mathrm{a}}$, Kay-Hooi Khoo ${ }^{\mathrm{a}}$, Yann Guerardel ${ }^{\mathrm{a}}$, Elisabeth Elass-Rochard ${ }^{\mathrm{a}}$

\begin{abstract}
Macrophage glycosylation is essential to initiate the host-immune defense but may also be targeted by pathogens to promote the infection. Indeed, the alteration of cell-surface glycosylation status may affect the binding of lectins involved in cell activation and adhesion. Herein, we demonstrate that infection by $M$. bovis BCG induces the remodeling of the $N$-glycomes of both human primary blood monocytes-derived macrophages (MDM) and macrophage-cell line THP1. MALDI-MS based $\mathrm{N}$-glycomic analysis established that mycobacterial infection induced increased synthesis of biantennary and multifucosylated complex type $\mathrm{N}$-glycans. In contrast, infection of macrophages by $M$. bovis BCG did not modify the glycosphingolipids composition of macrophages. Further nano-LC-MS ${ }^{n}$ glycotope-centric analysis of total $N$-glycans demonstrated that the increased fucosylation was due to an increased expression of the Le ${ }^{x}$ (Galß1-4[Fuc $\left.\alpha 1-3\right]$ GIcNAc) epitope, also known as stage-specific embryonic antigen-1. Modification of the surface expression of Le ${ }^{\mathrm{x}}$ was further confirmed in both MDM and THP-1 cells by FACS analysis using an $\alpha 1,3$-linked fucose specific lectin. Activation with the mycobacterial lipopeptide Pam3Lp19, an agonist of Toll-Like Receptor 2, did not modify the overall fucosylation pattern, which suggests that infection process is required to modify surface glycosylation. These results pave the way toward the understanding of infection-triggered cell-surface remodeling of macrophages.
\end{abstract}

\section{Introduction}

The glycome of immune cells comprises a complex mixture of glycans carried on proteins and lipids that decorate the cell surface, or secreted $(1,2)$, and is fundamental to initiating proper host defense (3-5). It is now well-appreciated that the biological activities of various glycosylated immune molecules such as the immune receptors (Toll-like receptors, mannose-receptor), IgG antibodies, cytokines, integrins and ligands of selectins, are dependent on their glycosylation status (6-8). Significant glycomic changes are noted in a wide range of pathological states including cancer (3), inflammatory diseases (9), and bacterial infection (10,11), and often positively correlated with regulated expression of glycosyltransferases (GTs) genes. In particular, alterations in both the terminal capping and elongation of glycans may affect interaction of cells with host immune lectins, such as selectins and galectins that transduce a multitude of functional effects including modulation of cell activation, survival and migration, or microbial lectins that mediate adhesion of pathogen (12). In turn, bacteria may manipulate host glycosylation to their own benefit. Several of these microorganisms invade macrophages in which they multiply and further control numerous cell-signaling pathways including production of cytokines and reactive oxygen species, fusion of phagosomes with lysosomes, cell apoptosis and autophagy (13-15). Modulated gene expressions of galactosyltransferases, sialyltransferases, neuraminidases and fucosidases in macrophages exposed to Mycobacterium tuberculosis (Mtb) have been reported $(16,17)$. Alteration of sialylation was also detected in murine macrophages activated with purified mycobacterial cell wall glycolipids, such as trehalose-dimycolate (TDM) $(18,19)$. To more precisely define the glycosylation changes based on these findings, we have investigated by a mass spectrometry approach how $M$. bovis BCG may modulate the glycome of infected human primary blood monocytes-derived macrophages (MDM) and the macrophage-like THP-1 cells, both commonly served as relevant cell models to study immunomodulatory functions of infected phagocytes $(20,21)$. Our in depth analysis revealed a significant increased expression of terminal fucosylated epitope, Lex, carried on complex type N-glycans, while the glycosphingolipids (GSL) profiles remained largely unaffected.

\section{Experimental}

Cell culture:

Differentiated THP-1 cells:

Human monocytic THP-1 leukemia cells (ECACC $\left.{ }^{\circ} 88081201\right)$ were differentiated into macrophage-like cells with $20 \mathrm{nM}$ phorbol12-myristate-13-acetate (PMA) for 72 h, in RPMI-1640 medium supplemented with $10 \%$ heat-inactivated FCS, 2 mM L-glutamine and $20 \mu \mathrm{M} \beta$-mercaptoethanol, as previously described $(22,23)$. Non-adherent cells were removed by washing with Phosphate Buffered Saline (PBS).

Human primary blood monocytes-derived macrophages (MDMs): 
Human blood samples from healthy donors were collected at the local blood transfusion center (Etablissement Français du Sang, Lille). Peripheral blood mononuclear cells (PBMC) were isolated by Ficoll density gradient centrifugation (Lymphoprep, AbCys). Monocytes were purified from PBMC by immunomagnetic selection with magnetic beads coupled to CD14, according to the instructions of the manufacturer (Miltenyi Biotech). Monocyte preparations were more than $95 \%$ pure, as checked by flow cytometry. MDMs were obtained by incubating freshly isolated monocytes (106 cells $/ \mathrm{mL}$ ) in complete RPMI 1640, 10\% fetal calf serum (FCS) supplemented with $10 \mathrm{ng} / \mathrm{mL}$ of GM-CSF for 6 days, as previously described in literature (24). Fresh medium with GMCSF was added after 2 and 4 days.

\section{Mycobacterial cell culture}

M. bovis BCG (Pasteur strain) were obtained from Pasteur Institute (Lille, France). Mycobacterium were grown in Sauton medium on shaker at $37^{\circ} \mathrm{C}$. Sauton medium was prepared as described by Larsen and collaborators (25).

Infection and activation of macrophage

After 6 days of monocytes differentiation into $M_{0}$, macrophages adherent cells were infected with M.bovis BCG, at MOI 5:1 (5 mycobacteria per macrophage) or activated by $0,5 \mu \mathrm{g} / \mathrm{ml}$ of mycobacterial lipopeptide $\mathrm{PAM}_{3} \mathrm{Lp}_{19}$ and maintained in a humidified atmosphere with $5 \% \mathrm{CO}_{2}$ at $37^{\circ} \mathrm{C}$ for $48 \mathrm{~h}(26,27)$. The activation of differentiated THP-1 cells was performed in similar conditions.

\section{Flow cytometry analysis of cell surface markers during cell activation:}

The activation of both PMA THP-1 cells and MDM, by either BCG-infection or lipopeptide stimulation, was controlled, by measuring the cell surface antigens expression (CD80, CD40, CD54) in unstimulated and activated cells, by flow cytometry. First, cells were pre-incubated $20 \mathrm{~min}$ at $4^{\circ} \mathrm{C}$ with Fc-receptor blocking reagent (Innovex Biosciences, Richmond, CA, USA) to decrease the nonspecific binding of immunoglobulin to cells. Cell surface immunostaining was directly stained, for $40 \mathrm{~min}$ at $4^{\circ} \mathrm{C}$ in $\mathrm{PBS} / 0.1 \% \mathrm{BSA}$ with fluorescein-conjugated mouse anti-human CD80, anti-CD40 Abs or PE-conjugated mouse anti-CD54 (BD Biosciences, Le Pont de Claix, France). Both FITC- or PE-conjugated mouse isotype control IgG (BD Biosciences) was used as negative control. Data were monitored on a flow cytofluorimeter (FACSCalibur, BD Biosciences) and analyzed with the CellQuest software (Mountain View, CA, USA).

\section{Quantitation of pro-infllammatory cytokines secretion by ELISA:}

After $8 \mathrm{~h}$ or $24 \mathrm{~h}$ activation, culture supernatants were collected and analyzed for the detection of TNF- $\alpha$, and IL-1 $\beta$, respectively, by sandwich ELISA, according to the manufacturers' instructions (Ozyme S.A.). Cytokine concentrations were determined using standard curves obtained with recombinant human TNF- $\alpha$ or IL-1 $\beta$. Statistical significance was determined using the Student $t$ test, with GraphPad Prism 5 (only values of $p<0.05$ were considered to be significant).

\section{Glycolipids extraction and purification:}

Infected or uninfected cells were detached from T75 flasks with cell dissociation non-enzymatic solution and washed twice with PBS. Cells $\left(5 \times 10^{6}\right)$ were lyophilized and extracted three times with $\mathrm{CHCl}_{3} / \mathrm{CH}_{3} \mathrm{OH}(2: 1, v / v)$ and once by $\mathrm{CHCl}_{3} / \mathrm{CH}_{3} \mathrm{OH}(1: 2, v / v)$ using intermediary centrifugations at $2500 \mathrm{~g}$ for $20 \mathrm{~min}$. Combined supernatants were dried under a nitrogen stream, subjected to mild saponification in $0.1 \mathrm{M} \mathrm{NaOH}$ in $\mathrm{CHCl}_{3} / \mathrm{CH}_{3} \mathrm{OH}(1: 1, \mathrm{v} / \mathrm{v})$ at $37^{\circ} \mathrm{C}$ for $2 \mathrm{~h}$ and evaporated to dryness. Samples were reconstituted in $\mathrm{CH}_{3} \mathrm{OH} / 0.1 \%$ TFA in water $(1: 1, v / v)$ and applied to a reverse phase $\mathrm{C}_{18}$ cartridge (Waters, Milford, MA, USA) equilibrated in the same solvent. After washing with $\mathrm{CH}_{3} \mathrm{OH} / 0.1 \%$ TFA in water $(1: 1, v / v)$, GSL were eluted by $\mathrm{CH}_{3} \mathrm{OH}, \mathrm{CHCl}_{3} / \mathrm{CH}_{3} \mathrm{OH}(1: 1, v / v)$ and $\mathrm{CHCl}_{3} / \mathrm{CH}_{3} \mathrm{OH}(2: 1, \mathrm{v} / \mathrm{v})$. The elution fraction was dried under nitrogen stream prior to structural analysis.

\section{Release and purification of $\mathbf{N}$-glycans:}

Cells were resuspended by Triton X-100 extraction buffer ( $1 \%$ Triton X-100 in PBS buffer). To lyse cells, suspension was sonicated $30 \mathrm{~min}$. Debris and insoluble fraction were pelleted down by centrifugation at $13000 \mathrm{rpm}$ for $10 \mathrm{~min}$ at $4^{\circ} \mathrm{C}$, and supernatant was kept. $1 / 10$ volume of $0,1 \mathrm{M}$ dithiothreitol were added to a final concentration of $10 \mathrm{mM}$ and incubated at $37^{\circ} \mathrm{C}$ for 1 hour, followed by addition of $1 / 10$ volume of $0,5 \mathrm{M}$ iodoacetamide 1 hour in the dark at $37^{\circ} \mathrm{C}$. The reduced/alkyled glycoproteins were precipitated with $1 / 9$ volume of $100 \%$ trichloroacetic acid to final $10 \%$ at $-20^{\circ} \mathrm{C}$ for $30 \mathrm{~min}$. Pellet was precipitated by centrifuge at $13000 \mathrm{rpm}$ for $10 \mathrm{~min}$ at $4^{\circ} \mathrm{C}$, then the supernatant was discarded. Pellet was resuspended and washed with $1 \mathrm{~mL}$ of cold acetone, then centrifuged at $13000 \mathrm{rpm}$ for $10 \mathrm{mins}$ at $4^{\circ} \mathrm{C}$, and repeat this step three times. Sample was incubated with trypsin (Sigma-Aldrich) in $50 \mathrm{mM} \mathrm{NH}_{4} \mathrm{HCO}_{3}, \mathrm{pH} 8.4$, overnight at $37^{\circ} \mathrm{C}$. The reaction was quenched by boiling at $100^{\circ} \mathrm{C}$ for 5 min. $\mathrm{N}$-Glycans were released by $\mathrm{N}$-glycosidase $\mathrm{F}$ (BioLabs) digestion at $37^{\circ} \mathrm{C}$ for 1 day and $\mathrm{N}$-glycans and $\mathrm{O}$-glycopeptides were separated by $\mathrm{C}_{18}$ Sep-Pak chromatography. $\mathrm{C}_{18}$ Sep-Pak was equilibrated in $5 \%$ aqueous acetic acid and washed in the same solvent. Sample was loaded on the $\mathrm{C}_{18}$ Sep-Pak and the released $\mathrm{N}$-glycans were eluted with $5 \%$ aqueous acetic acid and the bound peptides with $20 \%, 40 \%$ and $60 \%(v / v)$ propanol in $5 \%$ aqueous acetic acid, pooled and lyophilized.

Mass spectrometry analysis of Glycans: 
Glycans and glycolipids were permethylated according to the method of Ciucanu and Kerek (28) prior to mass spectrometry analysis. Briefly, samples were incubated with $\mathrm{DMSO} / \mathrm{NaOH} / \mathrm{ICH}_{3}$ during $2 \mathrm{~h}$ under agitation. The derivatization was stopped by addition of water and the permethylated glycans were extracted in $\mathrm{CHCl}_{3}$ and washed at least seven times with water. Then, permethylated $\mathrm{N}$-glycans were purified on $\mathrm{C}_{18}$ Sep-Pak. Samples were washed with ACN/0.1\% TFA in water $(1: 9, v / v)$ and eluted with ACN/0.1\% TFA in water $(8: 2, v / v)$. Permethylated glycans were solubilized in $A C N / \mathrm{H}_{2} \mathrm{O}(1: 1, v / v)$ and mixed with $2,5-$ dihydroxybenzoic acid matrix solution $\left(10 \mathrm{mg} / \mathrm{mL}\right.$ dissolved in $\mathrm{ACN} / \mathrm{H}_{2} \mathrm{O}(1: 1, \mathrm{v} / \mathrm{v})$ and spotted on MALDI plate. MALDI-TOF and MALDI-TOF/TOF spectra were acquired using a 4800 TOF/TOF spectrometer (Applied Biosystems, Framingham, MA, USA) in reflectron positive mode. The laser was set at $337 \mathrm{~nm}$ and a frequency of $200 \mathrm{~Hz}$ with 5000 shots per spot was used for MS and MS/MS data acquisition.

Additional permethylated glycan samples were analyzed by nanoLC-MS/MS on an Orbitrap Fusion Tribrid system (ThermoFisher Scientific), using the same reverse phase C18 nanoLC conditions, instrument settings, $\mathrm{MS}^{2}$-product dependent $\mathrm{MS}^{3}$ data acquisition, processing and analysis methods described previously (29). To identify potential changes in $\alpha 2-3$ vs 2-6-sialylation, the released glycan samples were subjected to dimethylamidation prior to permethylation (30) and mapped against the nondimethylamidated samples first by MALDI-MS on a MALDI TOF/TOF 5800 system (AB Sciex) and then by nanoLC-MS/MS analyses. Dimethylamidation was performed by adding $25 \mu \mathrm{L}$ of freshly prepared reagent containing $250 \mathrm{mM}$ EDC, $500 \mathrm{mM}$ HOBt, and 250 $\mathrm{mM}$ dimethylamine in DMSO to the glycan samples and incubated at $60^{\circ} \mathrm{C}$ for $1.5 \mathrm{~h}$. After drying down, samples were cleaned up by passing through C18 Sep-Pak cartridges equilibrated in $5 \%$ acetic acid before subjected to permethylation.

\section{Detection of carbohydrate epitopes by flow cytometry:}

To detect the variation of fucosylated epitopes at the cell surface of activated macrophages, the binding of FITC-conjugated plants lectins, such as LTA (Lotus tetranologobus agglutinin) and UEA-I (Ulex europaeus I agglutinin), which have a specificity for $\alpha$-Lfucose. was investigated by flow cytometry $(31,32)$. Untreated and infected cells $(250000 /$ well) were incubated with each lectin at $15 \mu \mathrm{g} / \mathrm{ml}$, for $40 \mathrm{~min}$ at $4{ }^{\circ} \mathrm{C}$ in PBS with calcium and magnesium, containing $0.05 \%$ BSA which has been previously pretreated by sodium metaperiodate. Furthermore, the non-specificity binding of LTA and UEA-I to cells was evaluated in presence of $\alpha$ Lfucose. After washing, fluorescence analysis was performed on a FACSCalibur, as previously described above. The log of fluorescence intensities in arbitrary units is plotted against cell number. For each lectin, the ratio of mean fluorescence intensity (MFI) of stimulated cells versus MFI of unactivated cells, was determined from three independent experiments performed in triplicate, with MDMs obtained from three blood donors. Statistical significance between untreated and stimulated cells was determined, using the Student $t$ test. Values of $p<0.05$ were considered to be significant.

\section{Results \& Discussion}

PMA-treated THP-1 cells and human primary monocytes-derived macrophages (MDMs) were used as models of study because they are known to display similar phagocytic capacity toward $M$. bovis $B C G$, leading to up-regulation of expression of cell surface markers and pro-inflammatory cytokines secretion (20). Prior to glycomic analysis, we have checked that macrophages (PMA THP1 and MDMs) infected by $M$. bovis BCG or incubated with the lipopeptide exhibited a pro-inflammatory phenotype through the increased expression of cell surface antigens and increased production of TNF- $\alpha$ and IL1- $\beta$ (SIFigure 1).

GSL profiles - Glycosphingolipids were then extracted and purified by solid phase extraction (SPE) on $\mathrm{C}_{18}$ cartridge and analysed by MALDI-MS in permethylated forms based on the calculated composition of glycan ceramide moieties and the prior knowledge of biosynthetic pathways of human GSLs (Fig. 1A). The MS/MS fragmentation analyses show that neutral GSLs are composed of (iso)globosides (i)Gb3 (Gal $\alpha 1-3 / 4 G$ al $\beta 1-4 G l c \beta 1-C e r)$ and Gb4 (GalNAc $\beta 1-3 G a l \alpha 1-3 / 4 G a l \beta 1-4 G l c \beta 1-C e r)$, as well as neutral ganglioside GA1 substituted by major ceramides [d18:1-C16:0 (Cer*)] and [d18:1-C24:0 (Cer**)] (SIFigure 2C). Among sialylated GSLs, ganglioside GM3 (Neu5Aco2-3Gal $\beta 1-4 G l c \beta 1-C e r)$ is the major structure, but other mono-sialylated structures such GM1, GD3 and GD1a were also detected. Infection by $M$. bovis BCG and activation by the lipopeptide PAM - $P_{19}$, did not modify the GSL patterns of THP1 cells (Fig. 1B, SIFigure 2A and 2B). Compared to PMA THP-1, MDM exhibited a much simpler GSL profile, dominated by GM3 associated with [d18:1/C16:0 (Cer*)] and [d18:1/C24:1 (Cer**)] (Fig. 1C), with traces of GM1 in some batches of MDM. As observed for PMA THP-1, M. bovis BCG infection did not induce any modification of the GSL profiles of MDM (SIFigure 3).

N-glycan profiles - The $\mathrm{N}$-glycans isolated from THP-1 and MDM cells were similarly profiled by MALDI-MS analysis of permethylated derivatives (Fig. 2A and Fig. 3A). In both cell types, the lower mass range signals ( $\mathrm{m} / \mathrm{z} 1500$ to 2500 ) were dominated by high-mannose $\mathrm{N}$-glycans that represent more than $50 \%$ of the total $\mathrm{N}$-glycans. The remaining compounds were identified as complex-type $\mathrm{N}$-glycans differently sialylated, branched and fucosylated (Fig. 2A and Fig. 3A; sTable 1). The relative amounts of individual $\mathrm{N}$-glycans signals were calculated by integrating their intensities and dividing them by the cumulated intensities of all signals and integrated into different groups sharing similar features (Fig. 2B and Fig. 3B). After infection by M. bovis BCG, PMA THP1 and MDM did not show significant variation on the ratio of high mannose type versus complex type $N$-glycans. Similarly, the 
prevalence of sialylation among complex type $\mathrm{N}$-glycans in THP-1 and MDM was not significantly modified. To ascertain if there is any significant change in the $\alpha 2,3 / \alpha 2,6$-sialyl linkages, the $N$-glycans from MDM cells, were subjected to linkage-specific dimethylamide modifications prior to permethylation and MS analysis. Initial MALDI-MS profiling indicated that after dimethylamidation, most of the peaks assigned as sialylated complex type $N$-glycans were visibly accompanied by additional peaks at 13 u higher (SIFigure 4), consistent with the presence of $\alpha 2,6$-sialyl linkage (30). For signals carrying multiple sialic acids, multiple degrees of +13 u were observed showing variable degree of $\alpha 2,6$-sialylation (bottom panels SIFigure 4). However, in all cases, there is a substantial amount of sialylated peaks remain unaffected, which indicates that sialylation on the $N$-glycans of MDM comprises both $\alpha 2,3$ and $\alpha 2,6$ linkages. Based on the relative intensities of the in source-generated oxonium ions at $\mathrm{m} / z$ 825 and 838 (SIFigure $5 A$ ), it could be further inferred that there is more $\alpha 2,3$ than $\alpha 2,6$ linkages but, most importantly, their relative amount was not appreciably affected upon $M$. bovis BCG treatment (SIFigure 5B).

Increased fucosylation - Contrary to sialylation pattern, infection by $M$. bovis BCG induced reproducible variations (over four replicates of THP-1 cell cultures and three MDM donors) of antennae and fucosylation levels (Fig. 2B and Fig. 3B) on complex Nglycans. In MDM the proportion of mono- and multi-fucosylated $N$-glycans increases from 15 to $20 \%$ and from 12 to $17 \%$, respectively. MALDI-MS/MS analysis of selected compounds showed that fucose residues could be attached to both chitobiose core and LacNAc extensions (data not shown) of fucosylated $\mathrm{N}$-glycans. However, these analyses could not clearly establish how the fucosylation pattern was modified following infection. In order to further delineate the nature and the extent of the glycosylation changes, we subjected the samples isolated from MDM cells to a semi-quantitative glycotope-centric glycomic mapping of total $\mathrm{N}$-glycome (29). Zooming in on fucosylated complex type $\mathrm{N}$-glycans, it is clear that upon BCG treatment, not only were several mono-fucosylated peaks detected at slightly higher intensity than the non-fucosylated counterparts, but also those carrying a second Fuc $(\mathrm{m} / \mathrm{z} 2508,2795,2883,3244,3833,3419,3606)$ became more apparent (SI Figure5). The second Fuc was confirmed to be associated with terminal Hex-HexNAc by the oxonium ion at $m / z 638$, which was only produced by MALDI in source fragmentation from sample treated with BCG (Fig 4). The detection of the range of terminal glycotopes by means of MALDI in source prompt fragmentation and the notable increase in fucosylation were corroborated by LC-MS/MS analysis of the permethylated sample under acidic conditions. Focusing only on the biantennary $\mathrm{N}$-glycans that were better resolved, it is clear that those structures assigned as carrying 2 to 3 fucose residues were expressed at a higher level upon BCG treatment, after being normalized to the $\mathrm{Man}_{9}$-high mannose peak (Fig. 4). Their structures were further verified by manual examining their respective HCD-MS 2 spectra (not shown), which produced the diagnostic $\mathrm{MS}^{2}$ ion at $m / z$ 638, (Fuc $\left.\mathrm{Hex}_{1} \mathrm{HexNAc}^{+}\right), 825\left(\mathrm{NeuAc}_{1} \mathrm{Hex}_{1} \mathrm{HexNAc}^{+}\right)$ but not $m / z 999\left(\mathrm{NeuAc}_{1} \mathrm{Fuc}_{1} \mathrm{Hex}_{1} \mathrm{HexNAc}^{+}\right)$.

Increased Lex expression - To resolve further the isomeric Fuc $\mathrm{Hex}_{1} \mathrm{HexNAc}_{1}$ glycotopes, the data dependent mode of $\mathrm{MS}^{2}$ acquisition during the LC-MS/MS run was programmed to be coupled with product-dependent $\mathrm{MS}^{3}$, targeting the $\mathrm{MS}^{2}$ ion at $m / z$ 638. In other words, whenever $\mathrm{m} / \mathrm{z} 638$ was detected among the $\mathrm{MS}^{2}$ ions, it would be automatically taken through to an $\mathrm{MS}^{3}$ event on the fly. Lex would be unambiguously identified if $\mathrm{MS}^{3}$ ion at $\mathrm{m} / \mathrm{z} 432$ was detected. In addition to manually interpreting several of the $\mathrm{MS}^{2} / \mathrm{MS}^{3}$ spectra acquired, the entire range of distinctive glycotopes expressed in MDM with and without BCG treatment, and their relative abundance, were mapped out based on the summed intensities of their respective diagnostic $\mathrm{MS}^{2}$ ions (Fig. 4). The results indicated that Le ${ }^{\mathrm{x}}$ was indeed more represented among the terminal glycotopes after BCG treatment. So was the core fucose as represented by the diagnostic MS ${ }^{2}$ ion at $m / z 468$. Moreover, there was no significant amount of LacdiNAc or Ley/Le ${ }^{b}(m / z ~ 812)$, but a low level of sialyl fucosylated Hex-HexNAc $(m / z$ 999) and diLacNAc $(m / z$ 913) was detected.

The increased expression of cell-surface fucosylated epitope was further confirmed by flow cytometry using two fluorescent $\alpha 1,3-$ fucose specific LTA and $\alpha 1,2$-fucose specific UEA-I lectins $(31,32)$. In agreement with MS analyses that showed an increased Lex expression, LTA displays a higher binding on PMA-THP1 (x2.1) (Fig. 5A) and MDMs (x4.5) (Fig. 5B) after infection ( $p<0,001, n=3$ ). Inversely, UEA signal associated to cells did not show any modification following infection. This recognition is carbohydratedependent, since fucose inhibits the LTA binding to cells (Fig. 5 A \& B, peaks 3).

Functional relevance - We have shown that infection by $M$. bovis BCG modifies the $N$-glycan patterns of human macrophage PMATHP-1 cell line and primary MDM. Most notably, we observed a modification in the average number of antennae and an increase in the surface expression of Le ${ }^{x}$ antigen. On the contrary, activation by Pam $L_{3} \mathrm{p}_{19}$, an agonist of TLR2, increases the level of biantennary $\mathrm{N}$-glycans but does not alter their fucosylation. This subtle difference suggests that mycobacterium-driven changes are not restricted to a TLR-2 dependent pathway. Our results are in agreement with Li and collaborators who suggested that only terminal and subterminal fucosylation, but not core fucosylation (linked $\alpha 1,6)$, may be considered as a hallmark of M1 inflammatory macrophages (33). Moreover, the biosynthesis of GDP-Fuc nucleotide or its intracellular transport in macrophage could be modified upon BCG infection, as previously reported in inflammation, tumorigenesis or apoptosis processes (34,35). Indeed, $M$. bovis BCG may induce a partial apoptosis of macrophages (36). Fucosylated $N$-glycans also participate in tissue remodeling by reducing the activity of tissue inhibitor of metalloproteinases-1 (TIMP-1) (37). Le is a stage specific embryonic antigen-1 (SSEA-1) and a marker for human myeloid cells that contributes to leukocytes recruitment to inflammatory sites by selectins and interacts with the immune receptor DC-SIGN. Several reports established that along differentiation and maturation of phagocytes, a dynamic regulation in expression of $s \mathrm{~L}^{\mathrm{x}}$ and Le $\mathrm{L}^{\mathrm{x}}$ occurs, which may be due to transcriptional or post-translational 
changes of sialyltransferases and neuraminidases expression (38). Indeed, both differentiation of monocytic THP-1 cells into macrophages and the maturation of DC reduce sLe ${ }^{x}$ expression at the cell surface $(22,39)$. The sLe ${ }^{x}$ epitope, expressed preferentially on molecules of monocytes such as PSGL1, CD43 and CD44, mediates the recruitment and adhesion of circulating leukocytes to endothelium by interactions with $E$-selectin (38).

MS-analysis also revealed an increase in the proportion of bi-antennary $\mathrm{N}$-glycans upon $\mathrm{M}$. bovis BCG infection. LacNAc epitopes are regulators of immunity since they are preferential ligands for galectins, a family of lectins including 15 members with various fines specificities (40). Galectins exert multifunctional immunomodulatory effects by regulating phagocytosis, apoptosis and by inducing or suppressing cell effector functions. Among those, binding affinity of galectin 3 expressed by macrophages might be affected by the apparent modulations of antennae and fucosylation. Furthermore, mycobacterial lectins may also recognize glycoconjugates on macrophages and thus be involved in the adhesion and internalization of pathogens (41). Bacterial lectins could also potentially manipulate the immune response, by competing with the host lectins for the glycans present at the surface of immune cells. In particular, a ricin-type $\beta$-trefoil lectin, encoded by $R v 1419$ gene, has been detected in pleural effusions and granulomas of patients with active TB (42). Interestingly, Rv1419 modulates adhesion to murine and human derived macrophages and influences the intracellular growth of $M t b$ (43). Although its ligands remain unknown, it is expected to interact with Gal $\beta 1,4$ GIcNAc $\beta$ patterns present in the human $N$-glycans. We cannot exclude that the modification of $N$-glycans antenna in infected macrophages modulates the affinity of this lectin to cells.

\section{Conclusion}

In conclusion, we have directly identified and highlighted alterations in the $N$-glycome of human macrophages upon infection with $M$. BCG bovis, the most significant of which is the increased expression of Le epitope. Further research is clearly needed to identify the spectrum of glycoproteins carrying the Lex epitope and to determine to what extent glycosylation changes are driven by mycobacteria to promote infection or/and by host-response for protection. Changes in fucose metabolism could be analyzed by click-chemistry in BCG-infected macrophages using GDP-FucAz. The effect of the 6-alkinyl-fucose inhibitor on macrophages infection by Mycobacterium should also be investigated.

\section{Conflicts of interest}

There are no conflicts to declare.

\section{Acknowledgements}

The work was supported by the Ministère de l'Enseignement Supérieur et de la Recherche (to CD), by VisonnAIRR grant from Région Hauts-de-France (to DM), and an Academia Sinica Investigator Award grant AS-IA-105-LO2 (to KHK). We are grateful to Marlène Mortuaire for technical advice in cell culture. We are indebted to the Research Federation FRABio (Univ. Lille, CNRS, FR 3688, FRABio, Biochimie Structurale et Fonctionnelle des Assemblages Biomoléculaires) and to PAGés platform (http://plateformepages.univ-lille1.fr) for providing the scientific and technical environment conducive to achieving this work. We further acknowledge LC-MS/MS data acquisition at the Academia Sinica Common Mass Spectrometry Facilities for Proteomics and Protein Modification Analysis located at the Institute of Biological Chemistry, Academia Sinica, supported by Academia Sinica Core Facility and Innovative Instrument Project (AS-CFII-108-107).

\section{Abbreviations}

Dendritic Cell-Specific ICAM-Grabbing non-integrin (DC-SIGN), Glycosyltransferases (GTs), Glycosphingolipids (GSL), GranulocyteMacrophage Colony Stimulating Factor (GM-CSF), LTA (Lotus tetranologobus agglutinin) Mass spectrometry (MS), MonocytesDerived Macrophages (MDM), Mycobacterium (M.), Mycobacterium tuberculosis (Mtb), N-glycans (NG), Phorbol-12-Myristate-13Acetate (PMA), Toll-Like Receptors (TLR), Trehalose-DiMycolate (TDM), Sialic acid binding Ig-like Lectins (SIGLECS), Stage Specific Embryonic Antigen-1 (SSEA-1), UEA-I (Ulex europaeus I agglutinin)

\section{Notes and references}

1. Moremen KW, Tiemeyer M, Nairn AV. Vertebrate protein glycosylation: diversity, synthesis and function. Nat Rev Mol Cell Biol. juill 
2012;13(7):448-62.

2. Maccioni HJF, Quiroga R, Spessott W. Organization of the synthesis of glycolipid oligosaccharides in the Golgi complex. FEBS Lett. 6 juin 2011;585(11):1691-8.

3. Pinho SS, Reis CA. Glycosylation in cancer: mechanisms and clinical implications. Nat Rev Cancer. sept 2015;15(9):540-55.

4. Rabinovich GA, van Kooyk Y, Cobb BA. Glycobiology of immune responses. Ann N Y Acad Sci. avr 2012;1253:1-15.

5. Johnson JL, Jones MB, Ryan SO, Cobb BA. The regulatory power of glycans and their binding partners in immunity. Trends Immunol. juin 2013;34(6):290-8.

6. Semel AC, Seales EC, Singhal A, Eklund EA, Colley KJ, Bellis SL. Hyposialylation of integrins stimulates the activity of myeloid fibronectin receptors. J Biol Chem. 6 sept 2002;277(36):32830-6.

7. Su Y, Bakker T, Harris J, Tsang C, Brown GD, Wormald MR, et al. Glycosylation influences the lectin activities of the macrophage mannose receptor. J Biol Chem. 23 sept 2005;280(38):32811-20.

8. Kataoka H, Yasuda M, lyori M, Kiura K, Narita M, Nakata T, et al. Roles of N-linked glycans in the recognition of microbial lipopeptides and lipoproteins by TLR2. Cell Microbiol. juill 2006;8(7):1199-209.

9. Schulz BL, Sloane AJ, Robinson LJ, Prasad SS, Lindner RA, Robinson M, et al. Glycosylation of sputum mucins is altered in cystic fibrosis patients. Glycobiology. juill 2007;17(7):698-712.

10. Barel M, Harduin-Lepers A, Portier L, Slomianny M-C, Charbit A. Host glycosylation pathways and the unfolded protein response contribute to the infection by Francisella: Glycosylation and UPR in Francisella infection. Cellular Microbiology. déc 2016;18(12):1763-81.

11. Hare NJ, Lee LY, Loke I, Britton WJ, Saunders BM, Thaysen-Andersen M. Mycobacterium tuberculosis Infection Manipulates the Glycosylation Machinery and the N-Glycoproteome of Human Macrophages and Their Microparticles. J Proteome Res. 6 janv 2017;16(1):247-63.

12. Magalhães A, Marcos-Pinto R, Nairn AV, Dela Rosa M, Ferreira RM, Junqueira-Neto S, et al. Helicobacter pylori chronic infection and mucosal inflammation switches the human gastric glycosylation pathways. Biochim Biophys Acta. sept 2015;1852(9):1928-39.

13. Marakalala MJ, Martinez FO, Plüddemann A, Gordon S. Macrophage Heterogeneity in the Immunopathogenesis of Tuberculosis. Frontiers in Microbiology [Internet]. 23 mai 2018 [cité 9 oct 2018];9. Disponible sur:

https://www.frontiersin.org/article/10.3389/fmicb.2018.01028/full

14. Sampath P, Moideen K, Ranganathan UD, Bethunaickan R. Monocyte Subsets: Phenotypes and Function in Tuberculosis Infection. Front Immunol. 2018;9:1726.

15. Siqueira M da S, Ribeiro R de M, Travassos LH. Autophagy and Its Interaction With Intracellular Bacterial Pathogens. Frontiers in Immunology [Internet]. 23 mai 2018 [cité 9 oct 2018];9. Disponible sur:

https://www.frontiersin.org/article/10.3389/fimmu.2018.00935/full

16. Silver RF, Walrath J, Lee $\mathrm{H}$, Jacobson BA, Horton $\mathrm{H}$, Bowman MR, et al. Human alveolar macrophage gene responses to Mycobacterium tuberculosis strains H37Ra and H37Rv. Am J Respir Cell Mol Biol. avr 2009;40(4):491-504.

17. Kumar D, Nath L, Kamal MA, Varshney A, Jain A, Singh S, et al. Genome-wide analysis of the host intracellular network that regulates survival of Mycobacterium tuberculosis. Cell. 5 mars 2010;140(5):731-43.

18. Mercurio AM, Schwarting GA, Robbins PW. Glycolipids of the mouse peritoneal macrophage. Alterations in amount and surface exposure of specific glycolipid species occur in response to inflammation and tumoricidal activation. J Exp Med. 1 oct 1984;160(4):1114-25.

19. Afroun S, Tenu JP, Lemaire G. Modifications of glycosylation patterns in macrophages upon activation. Biochim Biophys Acta. 16 sept 1988;971(2):137-47.

20. Mendoza-Coronel E, Castañón-Arreola M. Comparative evaluation of in vitro human macrophage models for mycobacterial infection 
study. Pathog Dis. 2016;74(6).

21. Chanput W, Mes JJ, Wichers HJ. THP-1 cell line: An in vitro cell model for immune modulation approach. International Immunopharmacology. nov 2014;23(1):37-45.

22. Delannoy CP, Rombouts Y, Groux-Degroote S, Holst S, Coddeville B, Harduin-Lepers A, et al. Glycosylation Changes Triggered by the Differentiation of Monocytic THP-1 Cell Line into Macrophages. J Proteome Res. 6 janv 2017;16(1):156-69.

23. Tsuchiya S, Kobayashi Y, Goto Y, Okumura H, Nakae S, Konno T, et al. Induction of maturation in cultured human monocytic leukemia cells by a phorbol diester. Cancer Res. avr 1982;42(4):1530-6.

24. Ohradanova-Repic A, Machacek C, Fischer MB, Stockinger H. Differentiation of human monocytes and derived subsets of macrophages and dendritic cells by the HLDA10 monoclonal antibody panel. Clin Transl Immunology. janv 2016;5(1):e55.

25. Larsen MH, Biermann K, Jacobs WR. Laboratory maintenance of Mycobacterium tuberculosis. Curr Protoc Microbiol. août 2007;Chapter 10:Unit 10A.1.

26. Schromm AB, Reiling N, Howe J, Wiesmüller K-H, Roessle M, Brandenburg K. Influence of serum on the immune recognition of a synthetic lipopeptide mimetic of the 19-kDa lipoprotein from Mycobacterium tuberculosis. Innate Immun. août 2010;16(4):213-25.

27. Riendeau CJ, Kornfeld H. THP-1 cell apoptosis in response to Mycobacterial infection. Infect Immun. janv 2003;71(1):254-9.

28. Ciucanu I, Kerek F. A simple and rapid method for the permethylation of carbohydrates. Carbohydrate Research. août 1984;131(2):209-17.

29. Hsiao C-T, Wang P-W, Chang H-C, Chen Y-Y, Wang S-H, Chern Y, et al. Advancing a High Throughput Glycotope-centric Glycomics Workflow Based on nanoLC-MS ${ }^{2}$-product Dependent-MS ${ }^{3}$ Analysis of Permethylated Glycans*. Molecular \& Cellular Proteomics. déc 2017;16(12):2268-80.

30. Jiang K, Zhu H, Li L, Guo Y, Gashash E, Ma C, et al. Sialic acid linkage-specific permethylation for improved profiling of protein glycosylation by MALDI-TOF MS. Anal Chim Acta. 15 août 2017;981:53-61.

31. Sughii S, Kabat EA, Baer HH. Further immunochemical studies on the combining sites of Lotus tetragonolobus and Ulex europaeus I and II lectins. Carbohydr Res. 1 janv 1982;99(1):99-101.

32. Yan L, Wilkins PP, Alvarez-Manilla G, Do SI, Smith DF, Cummings RD. Immobilized Lotus tetragonolobus agglutinin binds oligosaccharides containing the Le(x) determinant. Glycoconj J. janv 1997;14(1):45-55.

33. Li J, Hsu H-C, Ding Y, Li H, Wu Q, Yang P, et al. Inhibition of Fucosylation Reshapes Inflammatory Macrophages and Suppresses Type II Collagen-Induced Arthritis: Inhibition of Fucosylation in Arthritis. Arthritis \& Rheumatology. sept 2014;66(9):2368-79.

34. Niittymäki J, Mattila P, Renkonen R. Differential gene expression of GDP-L-fucose-synthesizing enzymes, GDP-fucose transporter and fucosyltransferase VII. APMIS. août 2006;114(7-8):539-48.

35. Moriwaki K, Narisada M, Imai T, Shinzaki S, Miyoshi E. The effect of epigenetic regulation of fucosylation on TRAIL-induced apoptosis. Glycoconjugate Journal. oct 2010;27(7-9):649-59.

36. Wojtas B, Fijalkowska B, Wlodarczyk A, Schollenberger A, Niemialtowski M, Hamasur B, et al. Mannosylated lipoarabinomannan balances apoptosis and inflammatory state in mycobacteria-infected and uninfected bystander macrophages. Microbial Pathogenesis. juill 2011;51(1-2):9-21.

37. Kim HI, Saldova R, Park JH, Lee YH, Harvey DJ, Wormald MR, et al. The presence of outer arm fucose residues on the N-glycans of tissue inhibitor of metalloproteinases-1 reduces its activity. J Proteome Res. 2 août 2013;12(8):3547-60.

38. Silva Z, Tong Z, Guadalupe Cabral M, Martins C, Castro R, Reis C, et al. Sialyl Lewisx-dependent binding of human monocyte-derived dendritic cells to selectins. Biochemical and Biophysical Research Communications. juin 2011;409(3):459-64.

39. Julien S, Grimshaw MJ, Sutton-Smith M, Coleman J, Morris HR, Dell A, et al. Sialyl-Lewis(x) on P-selectin glycoprotein ligand-1 is 
regulated during differentiation and maturation of dendritic cells: a mechanism involving the glycosyltransferases C2GnT1 and ST3Gal I. J Immunol. 1 nov 2007;179(9):5701-10.

40. Vasta GR, Feng C, González-Montalbán N, Mancini J, Yang L, Abernathy K, et al. Functions of galectins as 'self/non-self'-recognition and effector factors. Pathog Dis. 31 2017;75(5).

41. Abhinav KV, Samuel E, Vijayan M. Archeal lectins: An identification through a genomic search. Proteins. janv 2016;84(1):21-30.

42. Nogueira L, Cardoso FC, Mattos AM, Bordignon J, Figueiredo CP, Dahlstrom P, et al. Mycobacterium tuberculosis Rv1419 encodes a secreted 13 kDa lectin with immunological reactivity during human tuberculosis. Eur J Immunol. mars 2010;40(3):744-53.

43. Bafica A, Morales S, Eto C, Souza N, Nogueira L, Riley L, et al. A mycobacterial lectin promotes bacilli adhesion to macrophages and influences pathogen growth during infection (INM3P.409). The Journal of Immunology. 1 mai 2015;194(1 Supplement).

44. Varki A, Cummings RD, Aebi M, Packer NH, Seeberger PH, Esko JD, et al. Symbol Nomenclature for Graphical Representations of Glycans. Glycobiology. déc 2015;25(12):1323-4. 


\section{Figures}

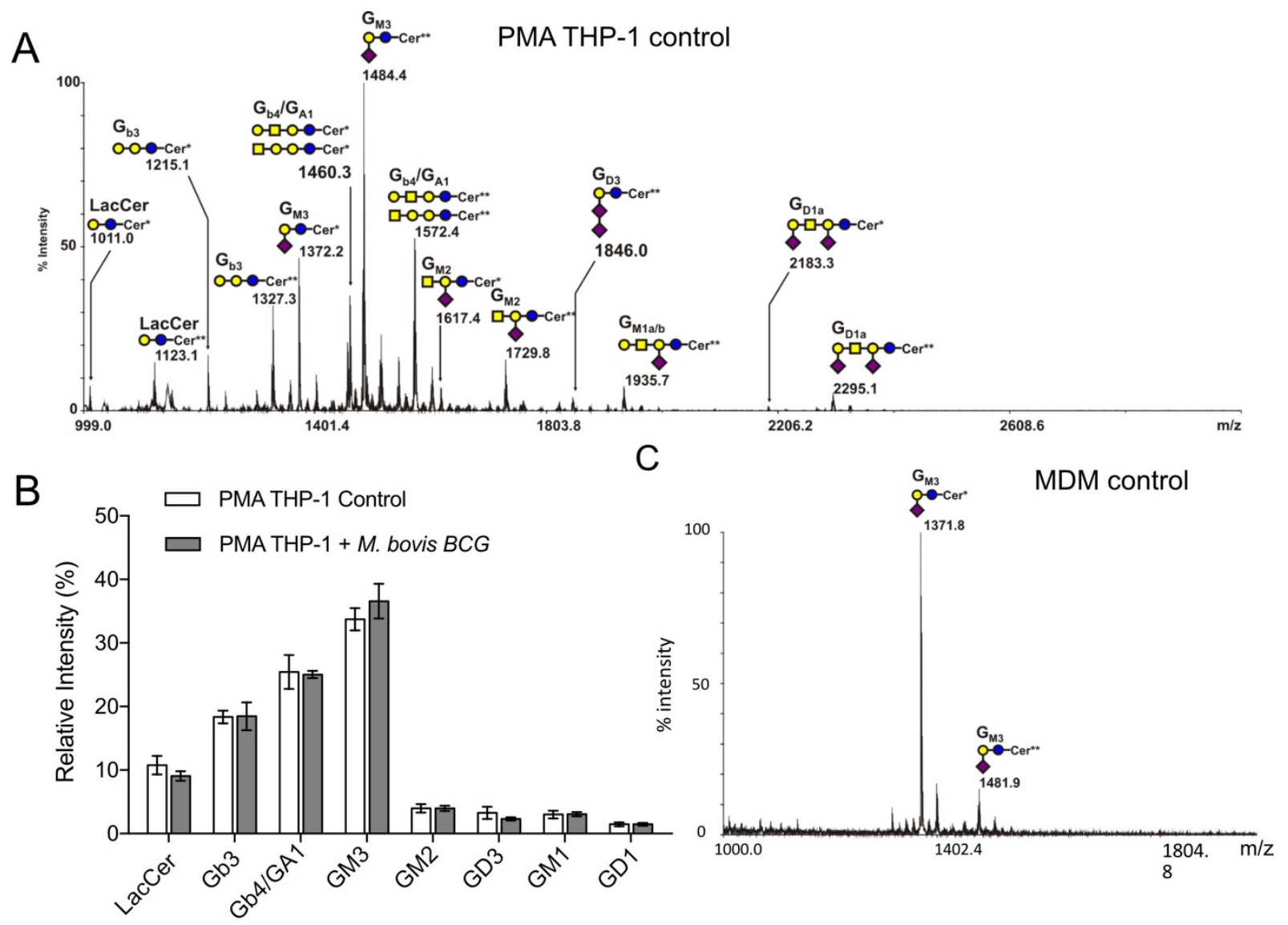

Figure 1: Mass spectrometry analysis of glycosphingolipids extracted from macrophages.

Representative MALDI-TOF-MS spectra (3 different experiments) of permethylated glycosphingolipids isolated from (A) PMA-THP-1 and (C) MDM cells. (B) relative quantifications of individual GLSs species extracted from PMA-THP-1 cells prior and after infection with M. bovis BCG. GSLs are present as $\mathrm{d} 18: 1 / \mathrm{C} 16: 0$ (Cer*) and d18:1/C24:0 (Cer**) isomers. Glycosyl composition assignment here was based on the detected mass values for the $[\mathrm{M}+\mathrm{Na}]^{+}$molecular ions and annotated using the standard Symbol Nomenclature for Glycan system (44). 


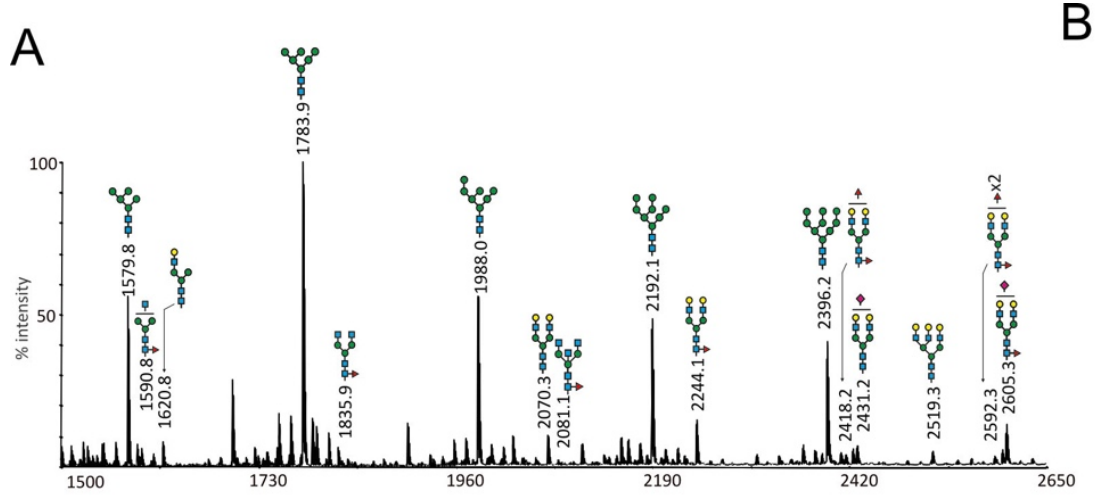

B
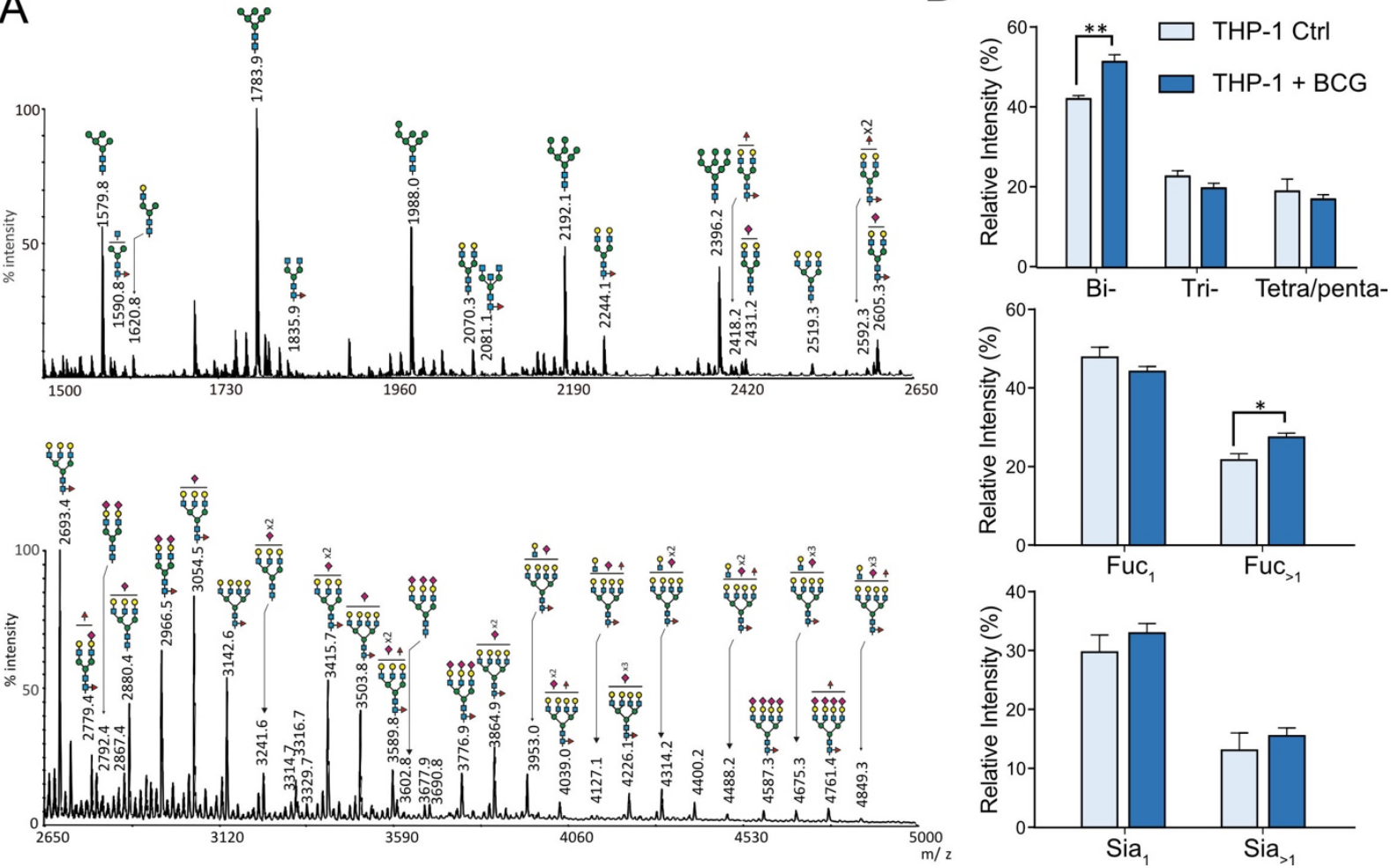

Figure 2: MALDI-MS analyses of $\mathrm{N}$-glycans isolated from THP1-cells. (A) MALDI-MS profile of permethylated N-glycans purified from uninfected PMA-differentiated THP-1 cells. The compositions of major glycan signals were deduced from the $m / z$ values of [M+Na] ${ }^{+}$adducts. Position of Fuc/Sia residues and branching patterns were not established at this stage and are only indicative. Complete list of identified $N$ glycans is provided in SITable 1. (B) Differential profiling of complex $\mathrm{N}$-glycans, extracted from PMA activated THP-1 cells, before (light blue) and after $M$. bovis BCG infection (deep blue). Top panel, proportions of multi-antennary complex-type $N$-glycans (bi, two LacNAc; tri, three LacNAc; tetra/penta, four and five LacNAc); middle panel, proportions of mono and multi-fucosylated complex type $N$-glycans; bottom panel, proportions of sialylated complex $\mathrm{N}$-glycan. Statistical significance compared with untreated cells $\left({ }^{* *} \mathrm{p}<0.01,\left({ }^{*} p<0.05\right)\right.$ was determined by a two-tailed t-test using GraphPad Prism 5 software. Data represent median \pm interquartile range from 4 independent experiments from different cell cultures. 


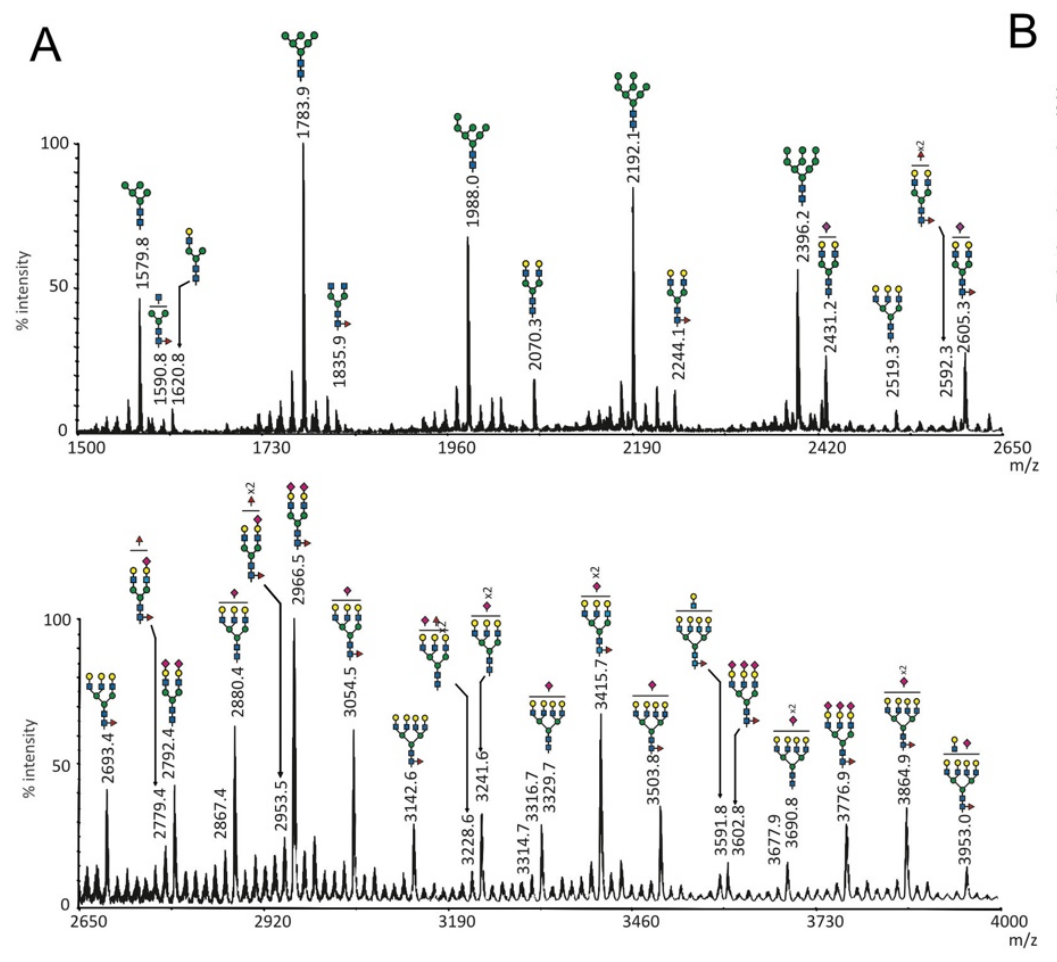

B
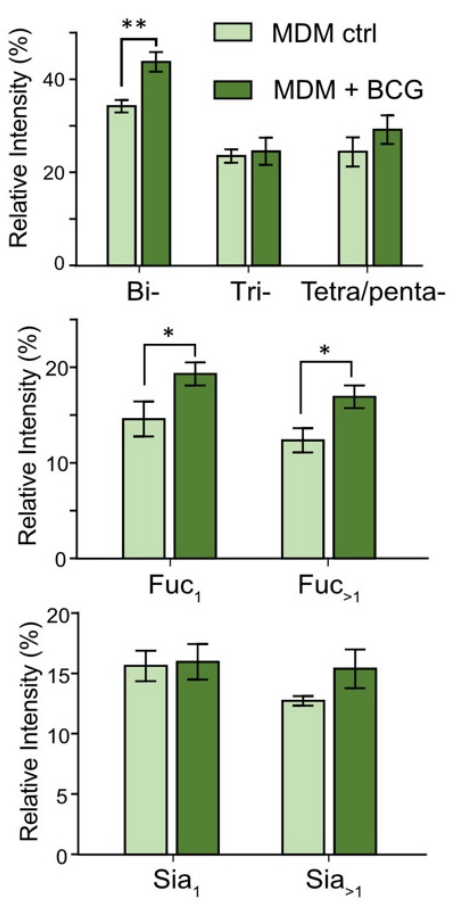

Figure 3: Mass spectrometry analysis of $\mathrm{N}$-glycans isolated from human MDM cells.

(A) MALDI-MS profile of permethylated $\mathrm{N}$-glycans purified from MDM cells. The composition of major glycan signals were deduced from the $\mathrm{m} / \mathrm{z}$ values of $[\mathrm{M}+\mathrm{Na}]^{+}$adducts. Position of Fuc/Sia residues and branching patterns were not established at this stage and are indicative. Complete list of identified $N$-glycans is provided in SITable 1. (B) Differential profiling of complex $N$-glycans before (light green) and after $M$. bovis BCG infection (dark green). Top, proportions of multi-antennary complex-type $N$-glycans (bi, two LacNAc; tri, three LacNAc; tetra/penta, four and five LacNAc); middle, proportions of mono and multi-fucosylated complex type $\mathrm{N}$-glycans; bottom, proportions of sialylated $\mathrm{N}$ glycan. Statistical significance compared with untreated cells $\left({ }^{* *} p<0.01,\left({ }^{*} p<0.05\right)\right.$ was determined by a two-tailed t-. Data represent median \pm interquartile range from three independent experiments with different donors. 
A

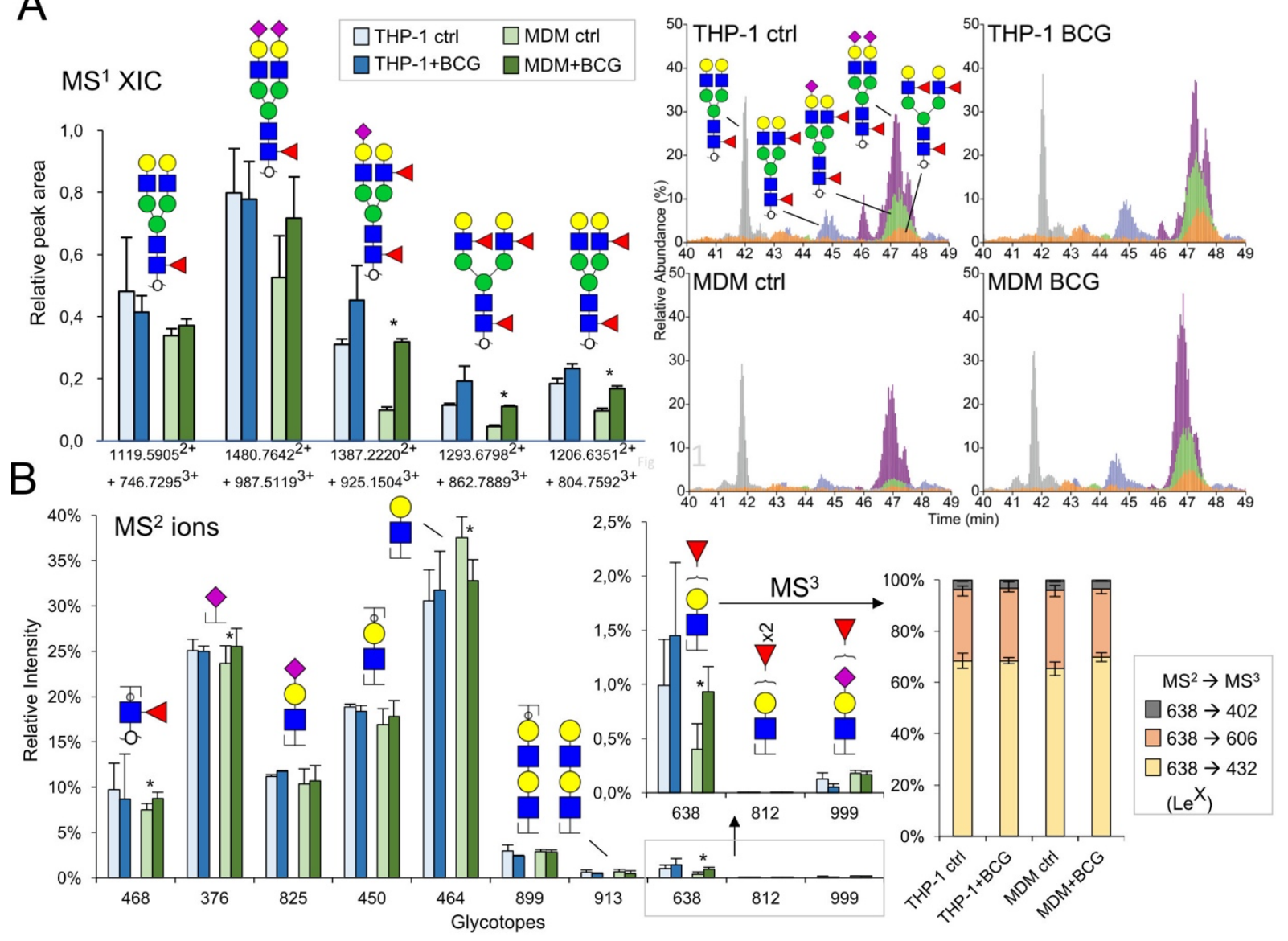

Figure 4. LC-MS/MS analysis of macrophages $\mathbf{N}$-glycans with and without BCG treatment. (A) The relative abundance of the five major sialylated and/or fucosylated biantennary $N$-glycans were inferred from extracted ion chromatogram plots (right panel, showing one set of data out of the three replicates) and relatively quantified based on their respective peak areas (left panel, averaged from 3 replicates, those of significance by statistical analysis were marked by *). The corresponding dataset from THP-1 cells were also plotted for side-by-side comparison). The relative peak areas were normalized to $\mathrm{Man}_{9}$ peak serving as internal reference (taken as 1.0 in the bar chart, and $100 \%$ in

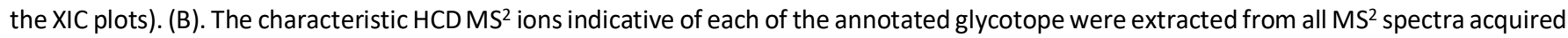
within the elution time of the $\mathrm{N}$-glycans identified. Their respective ion intensities were summed and then calculated as \% total for comparative analysis purpose only and not to be taken as true quantification. The charts for $\mathrm{m} / \mathrm{z} 638,812$ and 999 (boxed) were magnified to show clearly the increase in $\mathrm{m} / \mathrm{z} 638$ for the MDM dataset. The THP-1 dataset was similarly plotted for comparison. Despite the significant increase in $m / z 638$, the relative proportion of which that would yield $m / z 432$ by MS remained roughly constant. MS 3 ion at $\mathrm{m} / \mathrm{z} 606$ is indicative of $\mathrm{H}$ type 2 glycotope but may also be partly contributed by Lex. A small amount of MS ${ }^{3}$ ion at $m / z 402$ suggests that a very low level of Lea may also be present but the overall amount is negligible. 

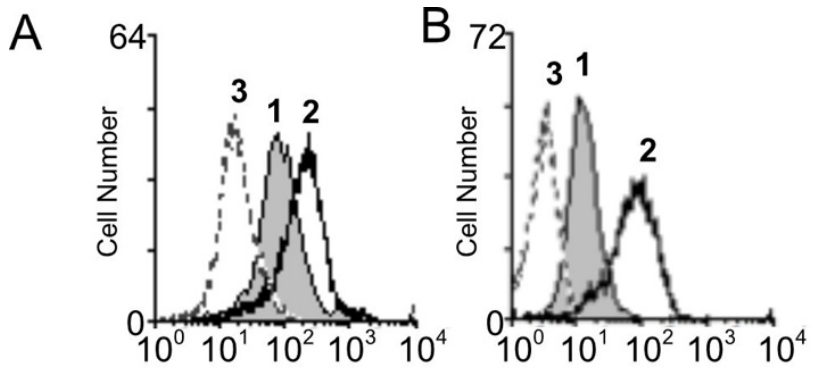

Fluorescence intensity Fluorescence intensity
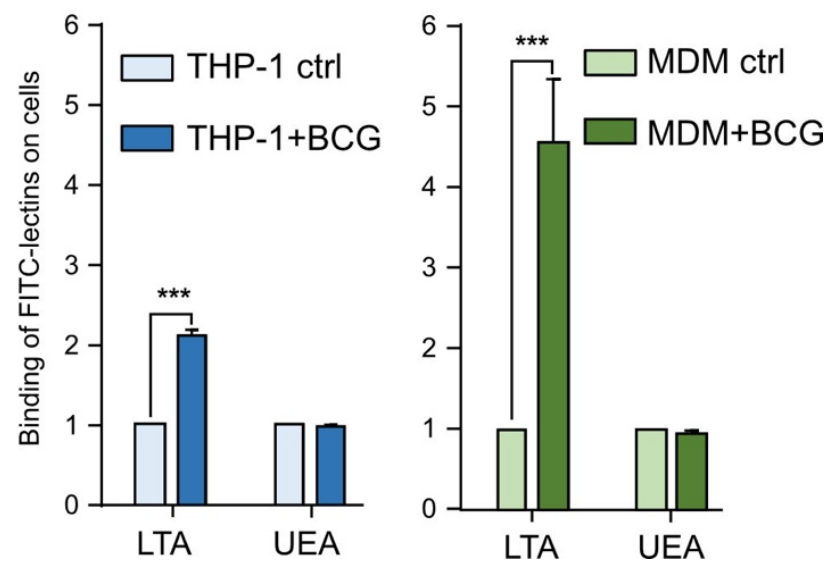

Figure 5. Binding of plant lectins to macrophages THP-1 and MDM, after infection by M. bovis BCG : (A) PMA-THP1 and (B) MDM were infected by $M$. bovis BCG for $48 \mathrm{~h}$ and washed with PBS. FITC-conjugated LTA or UEA-I lectins that interact specifically with $\alpha$ L-fucose, were incubated with untreated (ctrl) or stimulated cells, as described in "Method section". For each lectin, the mean fluorescence intensity (MFI) was determined by flow cytometry and the ratio MFI of infected cells versus MFI of untreated cells was determined. Binding of LTA lectin to unstimulated (grey peak 1) or to activated cells (peak 2) was illustrated in fluorescence histograms. The addition of L-fucose to cells inhibits the LTA interaction with activated cells (peak 3). These data shown are representative of three independent experiments with similar results $(* * * p<0,001)$. 